\title{
Structure of the dielectric tensor in nematic liquid crystals with topological charge
}

\author{
P. Vaveliuk, ${ }^{1,2, *}$ F. Moraes, ${ }^{3}$ S. Fumeron, ${ }^{4}$ O. Martinez Matos, ${ }^{1}$ and M. L. Calvo ${ }^{1}$ \\ ${ }^{1}$ Departamento de Óptica, Facultad de Ciencias Físicas, Universidad Complutense de Madrid, \\ Av. Complutense s/n, 28040 Madrid, Spain \\ ${ }^{2}$ Faculdade de Tecnologia, Servicio Nacional de Aprendizagem Industrial SENAI-Cimatec, \\ Av. Orlando Gomes 1845, 41650-010, Salvador, Bahia, Brazil \\ ${ }^{3}$ Departamento de Física, CCEN, Universidade Federal da Paraíba, cc 5008, 58051-970 João Pessoa, PB, Brazil \\ ${ }^{4}$ Laboratoire d'Énergétique et de Mécanique Théorique et Appliquée, CNRS UMR 7563, Nancy Université, \\ 54506 Vandœuvre Cedex, France \\ *Corresponding author: pablov@fisica.ufpb.br
}

Received January 5, 2010; revised April 16, 2010; accepted April 18, 2010; posted April 27, 2010 (Doc. ID 122736); published May 24, 2010

\begin{abstract}
A $q$-plate is a stratified medium composed of a uniaxial nematic liquid crystal with an inhomogeneous orientation of the optical axis possessing a topological charge $q$, which looks promising as a switching device. This work reports an approach to diagonalizing the dielectric tensor in $q$-plates, giving a detailed study of nontrivial tensor coordinate transformations. The relationship between the diagonal dielectric tensor and the topological charge of the plate is established. From that, the dielectric tensor for a given $q$-plate can be explicitly calculated in a convenient frame, and hence the field propagation equations can be derived. These studies establish a theoretical basis that could further deepen the research on these particular structures for nonconventional manipulation of the light. (C) 2010 Optical Society of America

OCIS codes: $000.3860,160.3710,260.1440,350.5500$.
\end{abstract}

\section{INTRODUCTION}

Recently, several works have been devoted to studying the wave propagation within stratified structures composed of a nematic liquid crystal possessing a topological charge [1-3]. From the observation of the behavior of such devices, called $q$-plates, one may consider that they can act as intermediaries for the spin-orbital angular momentum exchange of a monochromatic light beam [4]. The $q$-plates are promising devices, creating the potential for interesting applications such as switching devices such as polarization-spatial mode converters, for shaping the optical wavefront in a prescribed way, and for potential developments in optical communication and quantum computation $[5,6]$. In addition to $q$-plates used in these works, other space-variant optical elements, such as polarizers [7] and subwavelength gratings [8-10], help to deepen the analysis of space-variant polarization manipulation and wavefront-shaping manipulation of light through the Pancharatnam phase effect [8-11], generation of optical vortices of arbitrary topological charge, and nondifracting beams [7].

The formalism of the Helmholtz equation in these media is not a trivial one since $q$-plates have a topological defect that is a disclination of topological charge $q$. Besides, they are not only uniaxial but also optically inhomogeneous, wherein the optical axis locally varies its orientation $[4,12,13]$. The key point is to formulate correctly the dielectric tensor in these complex optical structures because, otherwise, an ill-posed dielectric tensor would lead to a nonphysical solution of Helmholtz's equation and, thereby, to a misleading interpretation about the optical properties of these devices. To the best of our knowledge, proper analyses on the structure of the dielectric tensor of arbitrary $q$-plates and on the connection between the topological charge and the local frame, wherein the dielectric tensor is diagonal, have not yet been performed. The works treating wave propagation in these structures, such as [1-3] in the paraxial and nonparaxial limits [14-16], were focused on the beam's angular momentum dynamics inside a particular kind of $q$-plate, namely, radial and azimuthal 1-plates. A general $q$ has been treated in [1]. However, no details on the derivation of the dielectric tensor are provided. Moreover, the relationship between the topological charge and the dielectric tensor has not yet been analyzed in structures with $q \neq 1$.

In this paper, we present a procedure to derive the dielectric tensor for an arbitrary $q$-plate based on the relationship between $q$ and the local frame, and we thereby calculate such a tensor in rectangular, circularcylindrical, and parabolic-cylindrical frames for several $q$-plates of interest. We emphasize that the procedure used in deriving the local frame for a given $q$-plate was established from the single knowledge of their topological charge $q$. This allows one to obtain the change-of-basis matrix between the local frame and any rectangular one. From this matrix, propagation field equations can be derived in the local frame for an arbitrary $q$-plate. The paper is structured as follows: Section 2 describes the main characteristics of the $q$-plate based on its topological charge. Section 3 is devoted to deriving the dielectric tensor of a $q$-plate for a given reference frame from the relationship among local and rectangular frames. In Section 
4 , the local frame for a given $q$-plate is constructed from knowledge of the topological charge. Finally, Section 5 gives concluding remarks.

\section{TOPOLOGY OF $q$-PLATES}

The $q$-plates described in [4] are planar layers (parallel to the $x y$ plane) of a nematic liquid crystal with a topological defect along the $z$ axis. A liquid crystal represents a special intermediate state of matter that simultaneously embodies the fluidity of a liquid and the anisotropy of a crystal. Substances that possess a nematic phase, one of the numerous types of liquid crystal phases, are characterized typically by an elongated rod-like molecular geometry. Unlike a solid crystal, whose molecules are rigidly locked into a global lattice structure, the individual molecules of a liquid crystal are free to move translationally as a fluid. The liquid molecules retain a long-range, orientational order with other molecules within the crystal. The $q$-plate is characterized by a unit vector, namely, the director $\hat{\mathbf{n}}(\mathbf{r})$ that describes the local (average) spatial orientation of non-chiral molecules. The fact that $\hat{\mathbf{n}}(\mathbf{r})$ and $-\hat{\mathbf{n}}(\mathbf{r})$ describe undistinguishable states is stated by saying that $\hat{\mathbf{n}}(\mathbf{r})$ is a headless vector. Ignoring Brownian fluctuations, when $\hat{\mathbf{n}}(\mathbf{r})$ is not defined at a certain point $\mathbf{r}_{s}$ (or at a succession of points), due to the distortions in the direction of $\hat{\mathbf{n}}$ there exists a topological defect in the nematic liquid crystal at the point $\mathbf{r}_{s}$. This can be formed naturally, formed as a result of phase transition processes, or artificially induced in the laboratory. The spatially inhomogeneous distribution of the optical axis is a remarkable feature of a $q$-plate. The optical axis lies in the direction of $\hat{\mathbf{n}}$, parallel to the $x y$ plane, and its orientation at each point $(x, y)$ can be represented in the continuum limit by the director lines, i.e., lines traced by $\hat{\mathbf{n}}$. When the origin of the coordinates is placed on the topological defect, i.e., $\mathbf{r}_{s}=(0,0)$, the director lines can be described by a scalar field, $\psi(x, y)$, representing the angle formed by $\hat{\mathbf{n}}$ with the $x$ axis [12,13] (see Fig. 1). This field is a linear function of the azimuthal coordinate $\varphi$ $=\arctan (y / x)$, and it depends on $q$ and on the angle $\psi_{0}$. The latter is the angle between the optical axis and the $x$ axis at $\varphi=0$. Explicitly, the relationship is $\psi=q \varphi+\psi_{0}$ $[12,13]$. The director vector now has the explicit form $\hat{\mathbf{n}}(\mathbf{r})=\hat{\mathbf{x}} \cos \psi+\hat{\mathbf{y}} \sin \psi$. Therefore, the director lines can be explicitly found as the solutions of

$$
d y / d x=\sin \left(q \varphi+\psi_{0}\right) / \cos \left(q \varphi+\psi_{0}\right) .
$$

Hence, the $q$-plate is fully characterized by $\left[q, \psi_{0}\right]$ rather

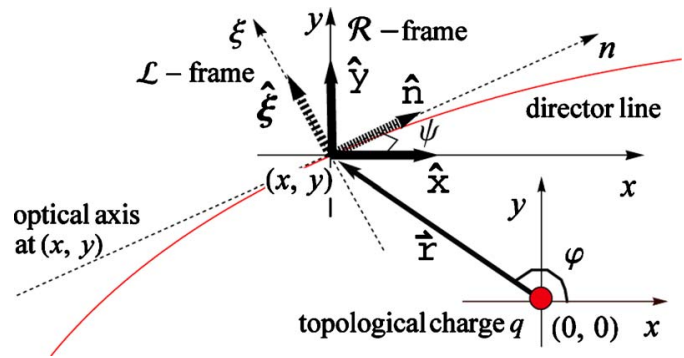

Fig. 1. (Color online) Relationship between the local frame and the rectangular one at point $(x, y)$ contained in a director line in any plane $z=z_{0}$. than just by $q$. Several geometries of interest represented by its director lines were derived and are depicted in Fig. 2.

\section{DIELECTRIC TENSOR IN $q$-PLATES}

The time-independent complex amplitude $\mathbf{E}(\mathbf{r})$ of a monochromatic wave field $\mathbf{E}(\mathbf{r}) e^{i \omega t}$ propagating inside a $q$-plate must satisfy the set constituted by the vector Helmholtz equation and the divergence Maxwell equation, including the dielectric tensor $\overline{\boldsymbol{\epsilon}}$. These yield

$$
\begin{gathered}
\nabla^{2} \mathbf{E}-\boldsymbol{\nabla}(\boldsymbol{\nabla} \cdot \mathbf{E})+k_{0}^{2-} \bar{\epsilon} \mathbf{E}=0, \\
\boldsymbol{\nabla} \cdot \overline{\boldsymbol{\epsilon}} \mathbf{E}=0 .
\end{gathered}
$$

The form of the $3 \times 3$ matrix representing $\overline{\boldsymbol{\epsilon}}$ depends both on the spatial distribution of the optical axis and on the reference frame in which the propagation problem is analyzed. Depending on both aspects, the matrix may have several possible structures:

1. $\overline{\boldsymbol{\epsilon}}$ will be diagonal only when the curves traced by the director lines (optical axis) on a given plane $z=z_{0}$ run either along or perpendicular to the curves traced by one of the coordinates of the frame used in the analysis. In this case, such a frame is named the local frame (see Fig. $1)$.

2. $\overline{\boldsymbol{\epsilon}}$ will be nondiagonal but independent of the coordinates when the curves traced by the director lines are geometrically identical to the curves described by one of the coordinates of the local frame but rotated at a given angle with respect to them. (see Fig. 2(a)).

3. $\overline{\boldsymbol{\epsilon}}$ will be not only nondiagonal but also dependent on the coordinates when the curves traced by the director lines are not identical to the curves traced by the coordinates of the local frame. The degree of difficulty in solving Eqs. (2) will depend strongly on the structure of the dielectric tensor. It is expected that these equations will possess the simplest form for a diagonal dielectric tensor, which implies the use of the local frame. But the task in identifying the local frame, even if it is feasible, is not, in general, straightforward. We shall undertake this task in the Section 4. In the present section, we shall standardize the procedure for finding the dielectric tensor for an arbitrary $q$-plate in an arbitrary reference frame. For this, we use the direct relationship between the local frame, say, $\mathcal{L}$, and the rectangular frame, say, $\mathcal{R}$, as displayed in Fig. 1 . We define $\mathcal{L}$ by introducing a unit vector, $\hat{\xi}$ perpendicular to $\hat{\mathbf{n}}$ and $\hat{\mathbf{z}}$. Thereby, the principal axes of the crystal coincide, point to point, with the coordinate axes of $\mathcal{L}$, so that the dielectric tensor $\overline{\boldsymbol{\epsilon}}$ (with real components, if absorption is neglected) is diagonal in the $\mathcal{L}$ basis upon operating on a monochromatic field $\mathbf{E}_{\mathcal{L}}=E_{n} \hat{\mathbf{n}}+E_{\xi} \hat{\boldsymbol{\xi}}+E_{z} \hat{\mathbf{z}}$ that propagates along the $z$ axis. Their explicit form is $\overline{\boldsymbol{\epsilon}}_{\mathcal{L}}$ $=\operatorname{diag}\left(n_{e}^{2}, n_{o}^{2}, n_{o}^{2}\right)$ with $n_{o}, n_{e}$ being the ordinary and extraordinary refractive indices, respectively. Notice, on the other hand, that at each point $\left(x, y, z_{0}\right)$, the $\mathcal{L}$ basis elements $\hat{\mathbf{n}}, \hat{\boldsymbol{\xi}}, \hat{\mathbf{z}}$ are related to the $\mathcal{R}$ basis elements $\hat{\mathbf{x}}, \hat{\mathbf{y}}, \hat{\mathbf{z}}$ via a rotation by an angle $\psi$ such that 

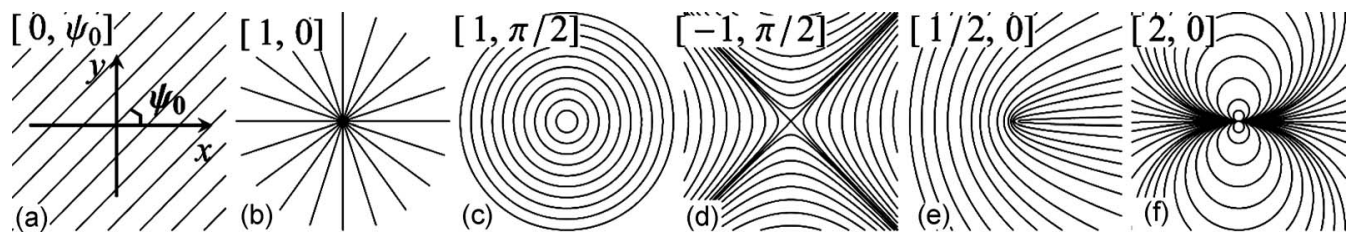

Fig. 2. Several allowed $q$-plate geometries of physical interest in an $x y$ plane fully characterized by $\left[q, \psi_{0}\right]$, where $q$ may be integer or half-integer. Notice that $q=0$ represents a plate with the optical axis homogeneously distributed and rotated by $\psi_{0}$ from the $x$ axis.

$$
\left(\begin{array}{c}
\hat{\mathbf{n}} \\
\hat{\boldsymbol{\xi}} \\
\hat{\mathbf{z}}
\end{array}\right)=\widetilde{\mathbf{R}}_{\psi}^{-1}\left(\begin{array}{c}
\hat{\mathbf{x}} \\
\hat{\mathbf{y}} \\
\hat{\mathbf{z}}
\end{array}\right),
$$

where $\widetilde{\mathbf{R}}_{\psi}$ is the usual counterclockwise rotation matrix around $\hat{\mathbf{z}}$ [17]. Hence, the dielectric tensor in the $\mathcal{R}$ frame, $\overline{\boldsymbol{\epsilon}}_{\mathcal{R}}$, depends on $\psi$, and it must be calculated from the diagonal tensor by means of a similarity transformation: $\overline{\boldsymbol{\epsilon}}_{\mathcal{R}}(\psi)=\widetilde{\mathbf{R}}_{\psi}^{-1} \overline{\boldsymbol{\epsilon}}_{\mathcal{L}} \widetilde{\mathbf{R}}_{\psi}$. Explicitly,

$$
\overline{\boldsymbol{\epsilon}}_{\mathcal{R}}(\psi(x, y))=\left(\begin{array}{ccc}
\frac{n_{+}^{2}+n_{-}^{2} \cos (2 \psi)}{2} & \frac{-n_{-}^{2} \sin (2 \psi)}{2} & 0 \\
\frac{-n_{-}^{2} \sin (2 \psi)}{2} & \frac{n_{+}^{2}-n_{-}^{2} \cos (2 \psi)}{2} & 0 \\
0 & 0 & n_{o}^{2}
\end{array}\right)
$$

where $n_{ \pm}^{2}=n_{e}^{2} \pm n_{o}^{2}$. It should be noted that Eq. (4) has physical meaning only if it operates on the field given in the rectangular frame, i.e., on $\mathbf{E}_{\mathcal{R}}=E_{x} \hat{\mathbf{x}}+E_{y} \hat{\mathbf{y}}+E_{z} \hat{\mathbf{z}}$. The first column of Table 1 gives the calculated $\overline{\boldsymbol{\epsilon}}_{\mathcal{R}}$ for some $q$-plates depicted in Fig. 2. $\overline{\boldsymbol{\epsilon}}_{\mathcal{R}}$ turns out to be diagonal for a homogeneous plate with null topological charge $(q=0)$ and with the optical axis either parallel or perpendicular to the $x$ axis: $\psi_{0}=0, \pi / 2$ [see Fig. 2(a)]. In fact, the director lines for both angles coincide with the coordinates of $\mathcal{R}$. Therefore, $\mathcal{R} \equiv \mathcal{L}$ for $q=0$ and $\psi_{0}=0, \pi / 2$. Any other $\psi_{0}$ preserves parallel straight lines as director lines but rotated by an angle $\psi_{0}$ with respect to the rectangular coordinates $(x, y)$. This yields a nondiagonal dielectric tensor, but with its elements independent of $(x, y)$. For an inhomogeneous $q$-plate $(q \neq 0)$, the structure of the dielectric tensor is quite complex in $\mathcal{R}$ since the nondiagonal matrices have, in addition, their elements dependent on $x, y$ because the director lines have different geometric form (no parallel straight lines) compared with the curves representing the $(x, y)$ coordinates, as Table 1 indicates. On the other hand, notice that Eq. (4) serves as a starting point to derive $\overline{\boldsymbol{\epsilon}}$ in an arbitrary curvilinear frame. In fact, we shall construct $\overline{\boldsymbol{\epsilon}}$ in any frame from $\overline{\boldsymbol{\epsilon}}_{\mathcal{R}}(\psi)$ by a change-ofbasis transformation. In a generic frame $\mathcal{A}$ characterized by the basis $\{\hat{\mathbf{a}}, \hat{\mathbf{b}}, \hat{\mathbf{z}}\}$, the dielectric tensor operating on the electric field $\mathbf{E}_{\mathcal{A}}=E_{a} \hat{\mathbf{a}}+E_{b} \hat{\mathbf{b}}+E_{z} \hat{\mathbf{z}}$ will be

$$
\overline{\boldsymbol{\epsilon}}_{\mathcal{A}}=\widetilde{\mathbf{M}}_{\mathcal{A}} \overline{\boldsymbol{\epsilon}}_{\mathcal{R}}(\psi) \widetilde{\mathbf{M}}_{\mathcal{A}}^{-1}=\widetilde{\mathbf{M}}_{\mathcal{A}} \widetilde{\mathbf{R}}_{\psi}^{-1} \overline{\boldsymbol{\epsilon}}_{\mathcal{L}} \widetilde{\mathbf{R}}_{\psi} \widetilde{\mathbf{M}}_{\mathcal{A}}^{-1},
$$

where $\widetilde{\mathbf{M}}_{\mathcal{A}}$ is the change-of-basis matrix between $\mathcal{R}$ and $\mathcal{A}$. Let us see some examples. We first analyze the circular-cylindrical frame, say, $\mathcal{C}$, with basis $\{\hat{\mathbf{r}}, \hat{\boldsymbol{\varphi}}, \hat{\mathbf{z}}\}$. The change-of-basis-matrix between $\mathcal{R}$ and $\mathcal{C}$ is a rotation matrix by an angle that is that determined by the azimuthal coordinate $\varphi$, i.e., $\widetilde{\mathbf{M}}_{\mathcal{C}} \equiv \widetilde{\mathbf{R}}_{\varphi}$. Care should be exercised not to confuse $\varphi$ with $\psi$. The dielectric tensor operating on the field $\mathbf{E}_{\mathcal{C}}=E_{r} \hat{\mathbf{r}}+E_{\varphi} \hat{\varphi}+E_{z} \hat{\mathbf{z}}$ is then given by $\overline{\boldsymbol{\epsilon}}_{\mathcal{C}}$ $=\widetilde{\mathbf{R}}_{\varphi} \widetilde{\mathbf{R}}_{\psi}^{-1} \overline{\boldsymbol{\epsilon}}_{\mathcal{L}} \widetilde{\mathbf{R}}_{\psi} \widetilde{\mathbf{R}}_{\varphi}^{-1}$. The second column of Table 1 gives the values for the calculated $\overline{\boldsymbol{\epsilon}}_{\mathcal{C}}$ from Eq. (5). As one could expect, $\overline{\boldsymbol{\epsilon}}_{\mathcal{C}}$ has turned out to be diagonal for the $[1,0]$ - and $[1, \pi / 2]$-plates depicted in Figs. 2(b) and 2(c). In fact, the radial and azimuthal director lines in these plates $(r$ $=$ constant and $\phi=$ constant, respectively) coincide with the coordinates of the circular-cylindrical frame, so that $\mathcal{C} \equiv \mathcal{L}$ for those geometries having $\hat{\mathbf{n}} \equiv \hat{\mathbf{r}}$ (or $\hat{\mathbf{n}} \equiv \hat{\boldsymbol{\varphi}}$ ) and $\hat{\xi}$ $\equiv \hat{\boldsymbol{\varphi}}$ (or $\hat{\xi} \equiv \hat{\mathbf{r}}$ ) for $[1,0]$ geometry (or $[1, \pi / 2]$ geometry). As previously mentioned, the propagation properties and applications in these geometries have been analyzed by various authors [1-6]. Finally, the third column in Table 1 gives the calculated $\overline{\boldsymbol{\epsilon}}$ in parabolic-cylindrical frame, say, $\mathcal{P}$, where the coordinates $(u, v, z)$ are defined by the transformations [18] $x=\left(u^{2}-v^{2}\right) / 2, y=u v$ and $z=z$, with $u$ $\in(-\infty, \infty), v \in(0, \infty)$. Use has been made of the basis $\{\hat{\mathbf{u}}$ $=\mu \hat{\mathbf{x}}+\nu \hat{\mathbf{y}}, \quad \hat{\mathbf{v}}=-\nu \hat{\mathbf{x}}+\mu \hat{\mathbf{y}}, \hat{\mathbf{z}}\} \quad$ with $\mu=u /\left(u^{2}+v^{2}\right)^{1 / 2}$ and $\nu$ $=v /\left(u^{2}+v^{2}\right)^{1 / 2}$. The change-of-basis matrix between $\mathcal{R}$ and $\mathcal{P}$, namely $\hat{\mathbf{M}}_{\mathcal{P}}$, is then constructed having as columns the vectors $\hat{\mathbf{u}}$, $\hat{\mathbf{v}}$, and $\hat{\mathbf{z}}$, so that one has $\overline{\boldsymbol{\epsilon}}_{\mathcal{P}}=\widetilde{\mathbf{M}}_{\mathcal{P}} \widetilde{\mathbf{R}}_{\psi}^{-1} \overline{\boldsymbol{\epsilon}}_{\mathcal{L}} \widetilde{\mathbf{R}}_{\psi} \widetilde{\mathbf{M}}_{\mathcal{P}}^{-1}$ operating on the field $\mathbf{E}_{\mathcal{P}}=E_{u} \hat{\mathbf{u}}+E_{v} \hat{\mathbf{v}}+E_{z} \hat{\mathbf{z}}$. By using Eq. (6), we obtain that the $[1 / 2,0$ (or $\pi / 2$ )]-plate, originally called disclination [19] and widely studied in the past $[12,13]$, has a diagonal dielectric tensor when analyzed in the $\mathcal{P}$ frame. In the latter, the coordinates coincide with the director lines of both $[1 / 2,0]$ and $[1 / 2, \pi / 2]$-plates. Without any additional quantitative analysis, we then can state that the director lines are two confocal parabolas having the $x$ axis as common axis. One opens into the positive $x$ axis ([1/2,0]-plate), and the other opens into the negative $x$ axis $([1 / 2, \pi / 2])$. The geometry $q=1 / 2$ holds great interest for applications, because it has recently been used as a geometric phase-plate in the spinorbital interaction of light in anisotropic subwavelength structures [20]. This would perhaps motivate a wave propagation analysis in these plates. Notice that analytic solutions for the scalar Helmholtz equation in terms of parabolic cylinder functions were reported for free-space propagation in the $\mathcal{P}$-frame [21], which could serve as a starting point for the full propagation problem in $[1 / 2,0(\pi / 2)]$-plates.

On the other hand, we verify that the geometries depicted in Fig. 2(d) and 2(f) $([-1, \pi / 2]$ and $[2,0])$ have a nondiagonal and coordinate-dependent dielectric tensor in the frames given in Table 1, as expected. However, as mentioned above, it is possible to diagonalize $\overline{\boldsymbol{\epsilon}}$ for these 
Table 1. Structure of the Dielectric Tensor $\bar{\epsilon}$ for the $q$-Plate Geometries $\left[q, \psi_{0}\right]$ Depicted in Fig. 2 in $\mathcal{R}, \mathcal{C}$, and $\mathcal{P}$ Frames ${ }^{a}$

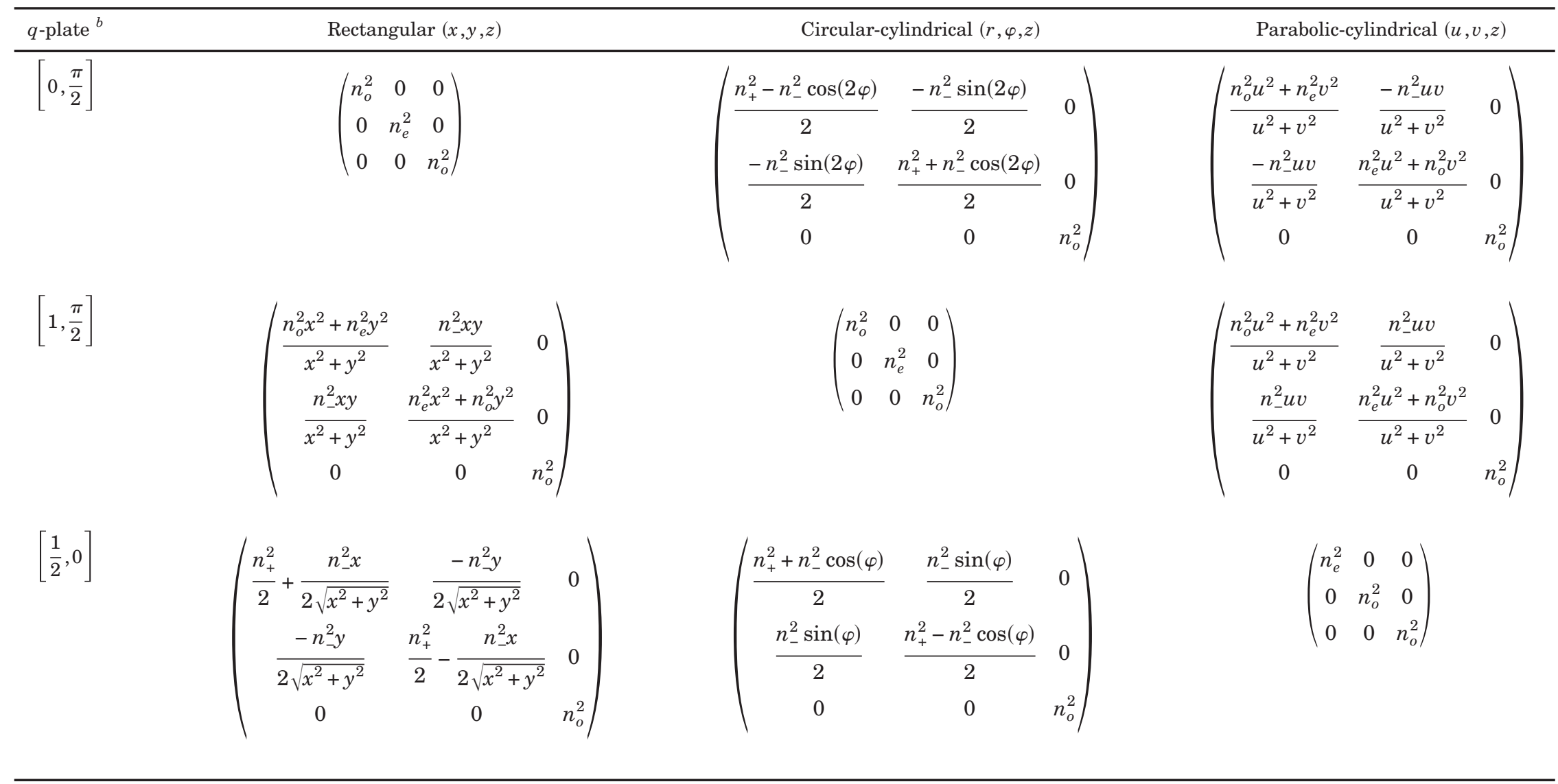

In the case of isotropic limit $n_{o} \equiv n_{e}=n$, all the matrices of this table reduce to $\bar{\epsilon}_{\text {ivo }}=n^{2} \operatorname{diag}(1,1,1)$, as expected.

${ }^{b}$ The matrices for $[q, 0]$ are obtained from the matrices for $\left[q, \frac{\pi}{2}\right]$ (or vice versa) by exchanging $n_{e}^{2} \leftrightarrows n_{o}^{2}$ in the first two rows 
particular structures and for any $\left[q, \psi_{0}\right]$-plate, in general, by constructing the corresponding local frame. This analysis will be performed in the next section.

\section{DERIVATION OF THE LOCAL FRAME FROM THE TOPOLOGICAL CHARGE}

In the previous section, we have given a general procedure to derive the dielectric tensor of an arbitrary $\left[q, \psi_{0}\right]$-plate in an arbitrary frame, from the relationship between the local and the rectangular frames. But this tensor will be diagonal only when the problem is formulated in the local frame. However, the geometrical complexity of some $q$-plates (see Fig. 2 , for example) makes it difficult to find the associated local frame. Fortunately, there exists a univocal connection between the local frame and the topological charge $q$ corresponding to the $q$-plate. By looking at the cases given in Table 1 , we have $q$ $=0 \leftrightarrows \mathcal{R}$-frame, $q=1 \leftrightarrows \mathcal{C}$-frame and $q=1 / 2 \leftrightarrows \mathcal{P}$-frame. We state that one can always choose a coordinate system where one of the coordinates runs along the director lines and the other one runs perpendicular to them. This leads us naturally to identify the dielectric permittivities parallel and perpendicular to the optical axis of the uniaxial medium. For the cases displayed in Table 1, we have found the relationship between $q$ and the $\mathcal{L}$-frame as follows:

- $q=0$. For $\psi_{0}=0$, the director components become $\hat{n}_{x}$ $=1$ and $\hat{n}_{y}=0$. By solving Eq. (1), the director lines are $x$ =cons. These lines, in turn, represent one of the coordinates of the rectangular frame. The other coordinate of this frame $(y=$ cons) can be found by solving Eq. (1) for $\psi_{0}=\pi / 2$. Finally, $\overline{\boldsymbol{\epsilon}}$ is diagonal in $\mathcal{R}$ for both $[0,0]$ and $[0, \pi / 2]$ structures [22].

- $q=1 / 2$. The director lines are explicitly given by $y /\left(-x+\left(x^{2}+y^{2}\right)^{1 / 2}\right)^{1 / 2}=u$ for $\psi_{0}=0$ and $\left(-x+\left(x^{2}+y^{2}\right)^{1 / 2}\right)^{1 / 2}$ $=v$ for $\psi_{0}=\pi / 2$. These generate two families of confocal parabolas ( $u=$ cons and $v=$ cons) having the $x$ axis as the common axis and one opening into the positive $x$ axis and the other into negative one. These two parabola families just coincide with the coordinates of the $\mathcal{P}$-frame.

- $q=1 . \psi_{0}=0$ generates radial straight lines ( $\left.r=c o n s\right)$ and $\psi_{0}=\pi / 2$, in turn, generates concentric circles $(\phi$ $=$ cons $)$, that are the coordinates of the $\mathcal{C}$-frame.
We then have the way to get the diagonal dielectric tensor by starting from the knowledge of the topological charge $q$. Therefore, we are able to derive the frames in which the dielectric tensor is diagonal for the other two geometries depicted in Fig. 2(d) $(q=-1)$ and 2(f) $(q=2)$. For the first case, the director components become $\hat{n}_{x}$ $=\sin \varphi$ and $\hat{n}_{y}=\cos \varphi$ for $\psi_{0}=\pi / 2$, and $\hat{n}_{x}=\cos \varphi$ and $\hat{n}_{y}$ $=-\sin \varphi$ for $\psi_{0}=0$. By solving Eq. (1) for both $\psi_{0}$ at a given plane $z=z_{0}$, we obtain two families of equilateral hyperbolas, with center at the origin. The first family arises from $x^{2}-y^{2}=2 \alpha=$ cons, having the straight lines $x=0$ and $y=0$ as the principal axes and $y=x$ and $y=-x$ as the asymptotes. The other family of equilateral hyperbolas is given by $x y=\beta=$ cons, having $y=x$ and $y=-x$ as the principal axes and $y=0$ and $x=0$ as the asymptotes. These orthogonal families of curves allow us to construct a frame $\mathcal{H}$ that, in the literature, is known as hyperbolic-cylindrical coordinates [23]. $\mathcal{H}$ is depicted in Fig 3(a), and it is defined by the coordinates $(\alpha, \beta, z)$ satisfying the following transformations:

$$
\begin{aligned}
& x= \pm \beta^{-1}\left(\alpha+\sqrt{\alpha^{2}+\beta^{2}}\right)\left(\sqrt{\alpha^{2}+\beta^{2}}-\alpha\right)^{1 / 2}, \\
& y= \pm\left(\sqrt{\alpha^{2}+\beta^{2}}-\alpha\right)^{1 / 2}, \\
& z=z
\end{aligned}
$$

with $\alpha, \beta, z \in(-\infty, \infty)$. The scale factors are then derived from Eq. (6) and given by

$$
\begin{aligned}
& h_{\alpha}=h_{\beta}=1\left[\sqrt{2}\left(\alpha^{2}+\beta^{2}\right)^{1 / 4}\right], \\
& h_{z}=1 .
\end{aligned}
$$

From Eqs. (6) and (7), one can derive the change-of-basis matrix between $\mathcal{R}$ and $\mathcal{H}$, say, $\tilde{\mathbf{M}}_{\mathcal{H}}$, as
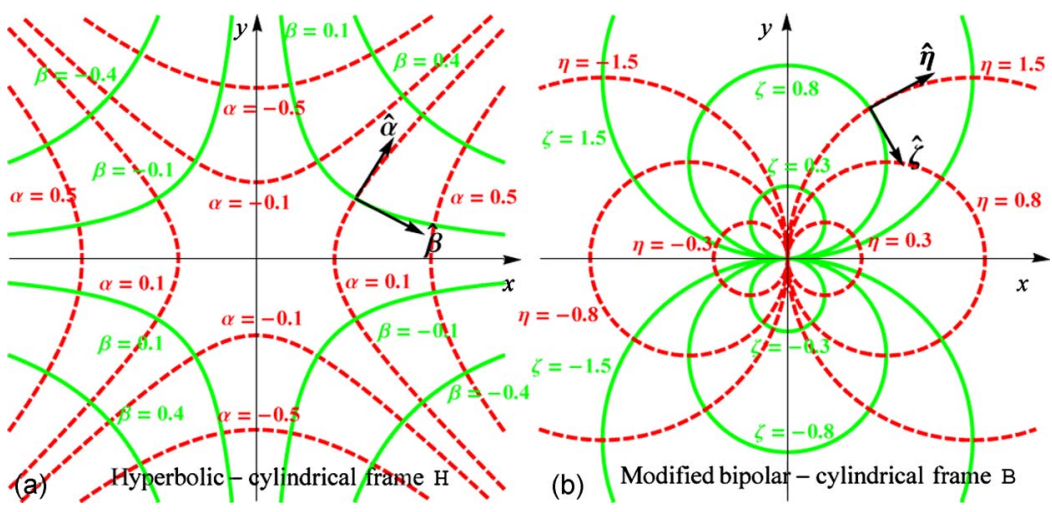

Fig. 3. (Color online) Traces of the coordinate surfaces on a plane $z=z_{0}$ : (a) for hyperbolic-cylindrical coordinates ( $\alpha=c o n s, \beta=c o n s$ ), related with $q=-1$, and $(b)$ for the modified bipolar-cylindrical coordinates $(\eta=$ cons, $\zeta=$ cons $)$, related with $q=2$. Dashed curves correspond to $\psi_{0}=\pi / 2$, and solid curves to $\psi_{0}=0$. 


$$
\tilde{\mathbf{M}}_{\mathcal{H}}=\left(\begin{array}{c}
\frac{\beta}{\sqrt{2 \sqrt{\alpha^{2}+\beta^{2}}\left(\sqrt{\alpha^{2}+\beta^{2}}-\alpha\right)}} \\
\frac{-\sqrt{\left(\sqrt{\alpha^{2}+\beta^{2}}\right)-\alpha}}{\sqrt{2}\left(\alpha^{2}+\beta^{2}\right)^{1 / 4}} \\
0
\end{array}\right.
$$

in which the columns represent the unit vectors $\hat{\boldsymbol{\alpha}}, \hat{\boldsymbol{\beta}}, \hat{\mathbf{z}}$ in the basis $\{\hat{\mathbf{x}}, \hat{\mathbf{y}}, \hat{\mathbf{z}}\}$. Hence, Eq. (8) allows us to find the dielectric tensor $\overline{\boldsymbol{\epsilon}}_{\mathcal{H}}$ operating in the field $\mathbf{E}_{\mathcal{H}}=E_{\alpha} \hat{\boldsymbol{\alpha}}+E_{\beta} \hat{\boldsymbol{\beta}}$ $+E_{z} \hat{\mathbf{z}}$ for any $q$-plate. Equations (6)-(8) provide us the full mathematical tool to derive the operators Div and Curl(Curl) and thereby to finally construct Eqs. (2) in the hyperbolic-cylindrical frame. In particular, we have verified that $\overline{\boldsymbol{\epsilon}}_{\mathcal{H}}=\operatorname{diag}\left[n_{e}^{2}\left(n_{o}^{2}\right), n_{o}^{2}\left(n_{e}^{2}\right), n_{o}^{2}\right]$, for $[-1, \pi / 2(0)]$ plates in $\mathcal{H}$.

For the case $q=2$, the director components become $\hat{n}_{x}$ $=-\sin 2 \varphi$ and $\hat{n}_{y}=\cos 2 \varphi$ for $\psi_{0}=\pi / 2$ and $\hat{n}_{x}=\cos 2 \varphi$ and $\hat{n}_{y}=\sin 2 \varphi$ for $\psi_{0}=0$. By solving Eq. (1), we obtain two sets of curves given by $y^{2}+x^{2}-\eta x=0$ and $y^{2}+x^{2}-\zeta y=0$ for $\eta$ $=$ cons and $\zeta=$ cons. These curves are Apollonian-like circles, i.e., two families of circles such that every circle in the first family intersects every circle in the second family orthogonally, and vice versa, which strongly suggests a bipolar-cylindrical frame [23]. However, the current bipolar-cylindrical frame as described in [23] is not appropriate for diagonalizing the dielectric tensor of the $q$-plate represented in Fig. 2(f). This structure appears to have null distance between the foci of the Apollonian circles, contrary to what happens with the conventional bipolar frame [23]. A bipolar system with null distance between the foci has not been studied previously in the scientific literature, to the best of our knowledge. We display it in Fig. 3(b) and refer to it as the modified bipolar-cylindrical frame, say, $\mathcal{B}$. At a given plane $z=z_{0}$, curves of constant $\eta$ correspond to non-concentric one-common-point circles $(x=0, y=0)$ of different radii $|\eta / 2|$, with centers located at $x<0, y=0$ for $\eta<0$ and at $x>0, y=0$ for $\eta>0$. The curves of constant $\zeta$ correspond to non-concentric one-commonpoint circles $(x=0, y=0)$ of different radii $|\zeta / 2|$, with centers located at $x=0, y<0$ for $\zeta<0$ and $x=0, y>0$ for $\zeta$ $>0$. These orthogonal circle families allow us to construct the $\mathcal{B}$-frame, defined by the coordinates $(\eta, \zeta, z)$, satisfying the following transformations:

$$
\begin{aligned}
& x=\eta \zeta^{2} /\left(\eta^{2}+\zeta^{2}\right), \\
& y=\eta^{2} \zeta /\left(\eta^{2}+\zeta^{2}\right), \\
& z=z,
\end{aligned}
$$

with $\eta, \zeta, z \in(-\infty, \infty)$. The scale factors, then, are given by

$$
\begin{aligned}
& h_{\eta}=\zeta^{2} /\left(\eta^{2}+\zeta^{2}\right), \\
& h_{\zeta}=\eta^{2} /\left(\eta^{2}+\zeta^{2}\right),
\end{aligned}
$$

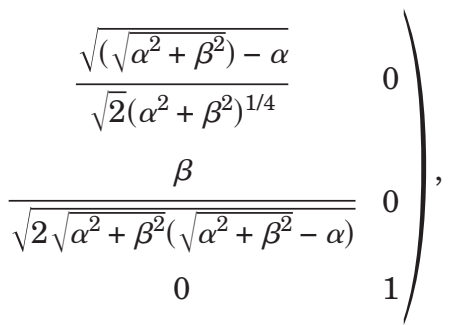

$$
h_{z}=1 \text {. }
$$

From Eqs. (9) and (10), one can derive the change-of-basis matrix between $\mathcal{R}$ and $\mathcal{B}$, say, $\widetilde{\mathbf{M}}_{\mathcal{B}}$, as

$$
\tilde{\mathbf{M}}_{\mathcal{B}}=\left(\begin{array}{ccc}
\frac{\zeta^{2}-\eta^{2}}{\eta^{2}+\zeta^{2}} & \frac{2 \eta \zeta}{\eta^{2}+\zeta^{2}} & 0 \\
\frac{2 \eta \zeta}{\eta^{2}+\zeta^{2}} & \frac{\eta^{2}-\zeta^{2}}{\eta^{2}+\zeta^{2}} & 0 \\
0 & 0 & 1
\end{array}\right)
$$

in which the columns represent the unit vectors $\hat{\boldsymbol{\eta}}, \hat{\boldsymbol{\zeta}}, \hat{\mathbf{z}}$ in the basis $\{\hat{\mathbf{x}}, \hat{\mathbf{y}}, \hat{\mathbf{z}}\}$, respectively. Equation (11) allows us to find the dielectric tensor $\overline{\boldsymbol{\epsilon}}_{\mathcal{B}}$ operating in the field $\mathbf{E}_{\mathcal{B}}$ $=E_{\eta} \hat{\boldsymbol{\eta}}+E_{\zeta} \hat{\zeta}+E_{z} \hat{\mathbf{z}}$ for any $q$-plate, and Eqs. (9)-(11) provide us all the tools to construct Eqs. (2) in the modified bipolar-cylindrical frame. Again, we verify that $\overline{\boldsymbol{\epsilon}}_{\mathcal{B}}$ $=\operatorname{diag}\left[n_{e}^{2}\left(n_{o}^{2}\right), n_{o}^{2}\left(n_{e}^{2}\right), n_{o}^{2}\right]$ for $[2, \pi / 2(0)]$-plates in $\mathcal{B}$. These two examples can be generalized in order to obtain the local frame with all their characteristic elements (transformations among this local frame and $\mathcal{R}$, scale factors, vector basis, change-of-basis matrix, differential operators, and so on) for any arbitrary $q$-plate. From this, it is possible to undertake the propagation problem in any of these complex structures. We remark that the analytical solution of Eqs. (2) and the physical interpretation of the results in the nonconventional but physically interesting structure $q=1 / 2$ are currently in progress by us. For brevity, results are not presented here. However, the search for the solutions for Eqs. (2) turns out to be quite difficult in these complex $q$-plates, even if the dielectric tensor is a diagonal one. We recall that other methods could help in seeking analytical field solutions in these complex $q$-plate geometries: for instance, the use of an effective metric, including the indices $n_{e}$ and $n_{o}$, within the Finsler geometry, which would transform a complex nondiagonal tensor into a diagonal one. The latter has been successfully employed in order to calculate light trajectories in nematic liquid crystals by using Fermat's principle [24].

\section{CONCLUDING REMARKS}

In summary, this work reports an approach to diagonalize the dielectric tensor in $q$-plates, giving a detailed study of nontrivial tensor coordinate transformations. Our method was used to calculate the structure of the dielectric tensor in Cartesian, circular-cylindrical, and paraboliccylindrical frames for several different $q$-plates. Furthermore, the procedure for deriving the local frame for a given $q$-plate was established from the single knowledge 
of its topological charge. This allowed one to obtain the change-of-basis matrix among these frames, local and rectangular ones. From that, field propagation equations can be derived in the local frame for an arbitrary $q$-plate. As an example, we obtain the local frames connected with the plates with $q=-1$ and with $q=2$, and their respective change-of-basis matrices. This procedure is useful since it establishes the theoretical basis for deepening the studies on this nonconventional (space-variant) manipulation of the light.

\section{ACKNOWLEDGMENTS}

P. V. and F. M. acknowledge financial support from $\mathrm{CNPq}$ (Brazilian federal grant agency). The partial financial support of the Spanish Ministry of Innovation and Science, under project TEC2008-04105is acknowledged.

\section{REFERENCES AND NOTES}

1. G. F. Calvo and A. Picón, "Spin-induced angular momentum switching," Opt. Lett. 32, 838-840 (2007).

2. E. Karimi, B. Piccirillo, L. Marrucci, and E. Santamato, "Light propagation in a birefringent -plate with topological charge," Opt. Lett. 34, 1225-1227 (2009).

3. P. Vaveliuk, "Nondiffracting wave properties in radially and azimuthally symmetric optical axis phase plates," Opt. Lett. 34, 3641-3643 (2009).

4. L. Marrucci, C. Manzo, and D. Paparo, "Optical spin-orbital angular momentum conversion in inhomogeneous anisotropic media," Phys. Rev. Lett. 96, 163905 (2006).

5. E. Karimi, B. Piccirillo, E. Nagali, L. Marrucci, and E. Santamato, "Efficient generation and sorting of orbital angular momentum eigenmodes of light by thermally tuned q-plates," Appl. Phys. Lett. 94, 231124 (2009).

6. E. Nagali, F. Sciarrino, F. De Martini, L. Marrucci, B. Piccirillo, E. Karimi, and E. Santamato, "Efficient generation and sorting of orbital angular momentum eigenmodes of light by thermally tuned $q$-plates," Phys. Rev. Lett. 103, 013601 (2009).

7. J. A. Ferrari, W. Dultz, H. Schmitzer, and E. Frins, "Achromatic wavefront forming with space-variant polarizers: Application to phase singularities and light focusing," Phys. Rev. A 76, 053815 (2007).

8. A. Niv, G. Biener, V. Kleiner, and E. Hasman, "Propagation-invariant vectorial Bessel beams obtained by use of quantized Pancharatnam-Berry phase optical elements," Opt. Lett. 29, 238-240 (2004).

9. Y. Gorodetski, G. Biener, A. Niv, V. Kleiner, and E. Has- man, "Space-variantpolarization manipulation for far-field polarimetry by use of subwavelength dielectric gratings," Opt. Lett. 30, 2245-2247 (2005).

10. A. Niv, G. Biener, V. Kleiner, and E. Hasman, "Manipulation of the Pancharatnam phase in vectorial vortices," Opt. Express 14, 4208-4218 (2006).

11. Q. Zhan and J. R. Leger, "Interferometric measurement of the geometric phase in space-variant polarization manipulations," Opt. Commun. 213, 241-245 (2002).

12. M. J. Stephen and J. P. Straley, "Physics of liquid crystals," Rev. Mod. Phys. 46, 617-704 (1974).

13. P. G. de Gennes and J. Prost, The Physics of Liquid Crystals (Oxford Univ. Press, 1993).

14. P. Vaveliuk, B. Ruiz, and A. Lencina, "Limits of the paraxial aproximation in laser beams," Opt. Lett. 32, 927-929 (2007).

15. P. Vaveliuk, G. F. Zebende, M. A. Moret, and B. Ruiz, "Propagating free-space nonparaxial beams," J. Opt. Soc. Am. A 24, 3297-3302 (2007).

16. P. Vaveliuk, "Quantifying the paraxiality for laser beams from the $M^{2}$-factor," Opt. Lett. 34, 340-342 (2009).

17.

$$
\widetilde{\mathbf{R}}_{\psi}=\left(\begin{array}{ccc}
\cos \psi & -\sin \psi & 0 \\
\sin \psi & \cos \psi & 0 \\
0 & 0 & 1
\end{array}\right) .
$$

18. P. M. Morse and H. Feshbach, Methods of Theoretical Physics, Part I (McGraw-Hill, 1953), pp. 501 and 658.

19. F. C. Frank, "On the theory of liquid crystals," Farad. Soc. Disc. 25, 19-28 (1958).

20. A. Niv, Y. Gorodetski, V. Kleiner, and E. Hasman, "Topological spin-orbit interaction of light in anisotropic inhomogeneous subwavelength structures," Opt. Lett. 33, 2910 (2008).

21. M. A. Bandres, J. C. Gutiérrez-Vega, and S. Chávez-Cerda, "Parabolic nondifracting optical wave fields," Opt. Lett. 29, 44-46 (2004).

22. For a given $q$, the alternative choices $\psi_{0}=0$ and $\psi_{0}=\pi / 2$ generate two families of orthogonal curves with the same geometry, each of them associated with the corresponding coordinates at the local frame. For $[q, 0]$, the dielectric tensor in its respective local frame is $\operatorname{diag}\left(n_{e}^{2}, n_{o}^{2}, n_{o}^{2}\right)$, and, for $[q, \pi / 2]$, it is $\operatorname{diag}\left(n_{o}^{2}, n_{e}^{2}, n_{o}^{2}\right)$. For any other $\psi_{0}(\neq 0, \pi / 2), \psi_{0}$ also generates an identical family of curves, but rotated in $\psi_{0}$ regardless the $x$ axis. There exists an exception: $q=1$.

23. K. Itõ and N. Sugakkai, Encyclopedic Dictionary of Mathematics (MIT Press, 1993), pp. 1727-1729.

24. C. Sátiro and F. Moraes, "Lensing effects in a nematic liquid crystal with topological defects," Eur. J. Phys. E 20, 173-178 (2006). 